Revue

de Sémantique

et Pragmatique
Revue de Sémantique et Pragmatique

38 | 2015

Le futur

\title{
Sur le prétendu figement des locutions conjonctives : le cas de lorsque et alors que
}

\section{Leïla Ben Hamad}

\section{(2) OpenEdition}

1 Journals

\section{Édition électronique}

URL : http://journals.openedition.org/rsp/931

DOI : $10.4000 /$ rsp.931

ISSN : 2610-4377

\section{Éditeur}

Presses universitaires d'Orléans

\section{Édition imprimée}

Date de publication : 1 septembre 2016

Pagination : 121-147

ISSN : 1285-4093

\section{Référence électronique}

Leilla Ben Hamad, «Sur le prétendu figement des locutions conjonctives : le cas de lorsque et alors que », Revue de Sémantique et Pragmatique [En ligne], 38 | 2015, mis en ligne le 01 février 2016, consulté le 14 avril 2020. URL : http://journals.openedition.org/rsp/931 ; DOI : https://doi.org/ $10.4000 /$ rsp.931

Ce document a été généré automatiquement le 14 avril 2020.

Revue de Sémantique et Pragmatique 


\title{
Sur le prétendu figement des locutions conjonctives : le cas de lorsque et alors que
}

\author{
Leïla Ben Hamad
}

\section{Introduction}

1 Selon la tradition lexicographique et grammaticale, les locutions conjonctives sont unanimement censées coder de façon univoque. Les différents éléments qui les composent sont comme fondus dans un moule unique. Un seul et même contenu sémantique se trouve ainsi confié à différentes locutions conjonctives, qui ne sont généralement pas en mesure de le coder ou le nuancent d'une façon spécifique en y greffant « un surplus » de contenu. Toutefois, nous ne pouvons souscrire à l'idée que toute locution conjonctive constitue un bloc figé, inanalysable, dont le sens rompt tout lien avec celui de ses constituants. L'objet de cette contribution est de montrer que chacune se présente, au contraire, comme une réalité stratifiée, dans laquelle se superposent deux types de contenus sémantiques : un contenu structurant servant de cadre général à la matière sémantique propre à la locution conjonctive et un contenu structuré formé des divers sèmes spécifiques aux éléments qui la constituent ${ }^{1}$. L'analyse que nous proposons tente de retrouver, derrière l'apparent figement ${ }^{2}$ des locutions conjonctives, la combinatoire sémantique qui les sous-tend. Cette combinatoire peut être mise concrètement en perspective par le biais d'une étude diachronique, qui doit aboutir in fine à délimiter les nuances sémantiques spécifiques que chaque locution conjonctive impose à la relation qu'elle établit ${ }^{3}$. La recherche se focalisera ici sur deux locutions conjonctives lorsque et alors que $e^{4}$, qui forment une paire, mais une paire moins bien connue et beaucoup moins décrite que lorsque et quand, alors que et tandis que. Nous nous attacherons à regarder du côté des propriétés mises en œuvre par les éléments qui les composent pour trouver un chemin d'accès à leur "sens global ${ }^{5}$ ». Nous examinerons, d'abord, la structure morpho-sémantique de chacune de ces locutions, qui n'a été jusqu'ici que superficiellement étudiée. Nous tenterons d'attribuer à 
chacune une identité spécifique cohérente avec celle, que nous aurons explicitée, de l'adverbe dont elle dérive ${ }^{6}$. Nous porterons aussi une attention particulière au mot que, qui entre dans leur composition. Nous essayerons d'entrer ensuite dans le domaine des faits de sens, de la variation du sens linguistique de ces formes, de leur rapport aux autres formes linguistiques, au monde et à l'esprit. Notre analyse à deux paliers permettra de mettre en relief un nouveau visage des locutions conjonctives, qui se démarque de la (ou les) face(s) qu'on lui prête habituellement ${ }^{7}$, et qui montre quels auxiliaires précieux elles représentent pour les opérations de connexion discursive.

\section{Structure morpho-sémantique de lorsque et alors}

\section{que}

\subsection{Les adverbes lors, alors}

2 Concernant les périodes les plus anciennes du français, il y a un point sur lequel s'accordent les grammaires historiques et les manuels aussi bien que les recherches les plus récentes : alors/lors, à l'opposé de or, marquent fondamentalement une référence dans le passé, à distance du moment de l'énonciation'. Lors et alors sont ainsi reconnus comme un co-domaine naturel du récit. ${ }^{9}$ À ce titre, c'est leur forme séquentielle qui est seule prise en compte. Il ne s'agit pas d'invalider les résultats mis au jour dans les différents travaux mentionnés ${ }^{10}$. Mais ils ne s'occupent pas ou peu de la spécificité sémantique des adverbes en question, ce qui ne peut éclairer notre appréhension du fonctionnement des locutions conjonctives qui en dérivent. La prise en compte de la compositionalité sémantique de lors et alors nous semble, encore une fois, primordiale ou première, dans la tentative d'en décrire les propriétés communes ou discriminatoires, l'ignorer serait risquer d'en fausser la description.

\subsubsection{L'origine de lors et alors}

3 Au regard de Wartburg (1992: 467-475), or, lors et alors ont le même étymon, le latin hora, 'heure' ${ }^{\prime 1}$. Observons cependant que, selon Imbs (1956: 209), lors est bâti non sur un nom temporel du latin mais sur un adverbe médiéval. Il voit dans le désir d'expressivité du locuteur le facteur déclenchant l'intégration de ce nouveau morphème dans la grammaire, ainsi que celle un peu plus tardive de alors ${ }^{12}$. Mais cette vue se heurte à la réalité des textes. Il faut la reconsidérer, ou tout au moins la complexifier. En effet, dans Libri psalmorum, où se présente une variante de lors, qui est de loin la plus fréquente :

(1)E sicume en la tue pramesse, lores serunt tresturnet li mien enemi ariere, En quelqueunques jurn apelerai tei ; astetei je conui que tu ies li miens Deus. (Libri psalmorum : 73 ; première moitié du XIIe siècle)

on trouve la construction l'+ ore, où ore se comporte comme un nom, comme en latin :

(2)E establi la tempested de lui en l'ore, e turent li fluet de lui. E esledecerent pur ce qu'il se turent, e demena els el port de la volunted d'els. (Libri psalmorum : 165 ; première moitié du XIIe siècle)

5 C'est à partir du nom heure, issu de hora latin, et de l'article défini, qui a pour origine le démonstratif latin illa (distal, ou de troisième personne) ${ }^{13}$, que lors s'est - semble-t-ildéveloppé ${ }^{14}$. Aussi ilores existe dès les plus anciens textes, à côté de lores :

(3)Que lores sunt alees 
Les hures e passees

Dunt nus le jur furmum

Que bisexte appellum. (Philippe de Thaon, Comput, v. 2095-2098 ; 1113 ou 1119)

(4)Ciel e terre crïat

Li reis ki nus furmat

Terre ert ilores vaine.

De tut en tut baraine. (Philippe de Thaon, Comput, v. 2003-2006, 1113 ou 1119)

Il s'ensuit que l'explication de l'émergence de ilores par aphérèse pré-littéraire ou préhistorique et renforcement d'un $-i$ analogique ne semble pas bien fondée. Le $i$ (ai ou a) initial n'est - semble-t-il - pas un apport postérieur pour qu'il soit considéré comme un affixe de renforcement. Le fait que alors soit bâti sur lors ne va pas de soi, non plus ${ }^{15}$. Issu de morphèmes latins, qui constituaient un syntagme nominal ${ }^{16}$, alors apparaît d'abord en ancien français comme un syntagme nominal aussi, formé d'une préposition et d'un nom, pour reproduire ou rendre fidèlement la construction latine, ou d'une préposition, d'un déterminant (un article, en l'occurrence ${ }^{17}$ ) et d'un nom, avant de ne devenir adverbe. Et c'est effectivement ce qu'atteste notre corpus ${ }^{18}$. Nous interrogerons, dans ce qui suit, le rapport de lors et alors avec leur origine. Nous nous emploierons à rendre compte de leur valeur intrinsèque, qui ressortit à la valeur de leurs composants.

\subsubsection{Propriétés communes aux deux adverbes}

7 Nous tenterons de dégager, ici, le noyau de sens commun à lors et alors. Notre analyse s'appuie - nous l'avons annoncé ci-dessus - sur la structure interne des deux adverbes. Deux questions seront examinées ici : la première concerne les particularités du lexème d'origine, le nom heure, la seconde traite de la valeur spécifique de l'article défini.

\section{a. Particularités du nom heure}

8 La nature du nom qui entre dans la composition de lors et alors prend part à la détermination des propriétés concernant leur fonctionnement. C'est d'un nom de temps qu'il s'agit, le substantif heure, issu du latin hora. Il désigne, comme le note Wartburg (op. cit. : 467), « la 24e partie du jour » :

(5)E l'ure de midi

Chantent clerc a midi (Philippe de Thaon, Bestiaire, v.267-268; entre 1121 et 1135)

Il sert aussi pour désigner le temps, le moment, une époque quelconque, indéterminée, comme on le voit dans l'exemple :

(6)(...) Et li cinquiesmes a vos muet

Tant con chevax porter le puet ;

Ne gart l'ore que il vos fiere ;

Li catre sont remés arriere,

Mes ne sont gaires de ci loing:

Tuit le secorront au besoing. (Chrétien de Troyes, Erec et Enide, v. 2987-2992 ; vers

1170)

Il peut avoir une extension référentielle vague. Cela paraît confirmé par les exemples suivants, où le nom heure est associé aux noms terme et tens :

(7)Quë il aveir volait

De cels que il mainteneit

A termë e a ure

(8)E senz tute demure (Philippe de Thaon, Comput, v.1883-1886; 1113 ou 1119) 
Li rois comanda aprester

Le souper, quant tans fu et ore. (Chrétien de Troyes, Erec et Enide, v.5532-5533;

vers 1170)

11 Au regard de ces configurations, heure peut avoir un comportement analogue à tens, terme, moment, dont le référent est un segment de temps non étalonné et d'extension instable $^{19}$. Cela permet justement à heure de donner lieu à un repérage de type anaphorique. Ce qui distingue par contre heure des autres noms, c'est qu'il appartient de façon intrinsèque au "référentiel chronologique», au sens de Berthonneau (notamment 1989 : 407-468) ${ }^{20}$ et qu'il porte de la sorte une valeur subjective métaphorique que les autres noms ne possèdent pas $^{21}$. Aussi s'agit-il d'un nom de mesure de temps, ce qui introduit la possibilité d'une métrique : on peut différencier, compter, ordonner des occurrences.

\section{b. L'article défini et ses valeurs}

L'opération sémantique que réalise l'article défini a suscité une abondante littérature ${ }^{22}$. Nous nous bornerons ici à ce qu'avaient écrit des historiens de la langue comme Foulet (1930 [19903]), Moignet (1973) et Buridant (2000) et aux études diachroniques menées sur l'ancien français, notamment Epstein (1995), Carlier \& Goyens (1998) et aussi Garnier (1999) ${ }^{23}$. Un consensus minimum se fait, dans ces différents travaux, sur les facteurs qui jouent un rôle crucial dans l'explication du sens et de la distribution de l'article défini. On mentionne tant soit peu "l'identifiabilité » du référent, sa mention préalable ou sa présence dans le contexte. Les divers emplois de l'article défini en ancien français peuvent converger, de ce point de vue dans le concept d'anaphorique, que reflète bien avant tout l'étymologie latine. Ainsi Buridant (2000) : 120) écrit :

"L'article défini peut avoir la valeur démonstrative héritée de ille et fonctionner comme article démonstratif en reprise anaphorique $»^{24}$

Epstein (1995 : 58-71) va plus loin. Il y a, selon lui, « des facteurs expressifs», qui doivent être soulignés, tels que :

«(...) la thématicité du référent, sa focalisation ou son importance par rapport à

d'autres référents de la même catégorie. »

Outre la fonction référentielle, l'article défini possède, à son sens, une fonction expressive, d'autant qu'il peut indiquer « la proéminence d'un référent »:

« (...) l'article défini en AF s'emploie d'une manière expressive quand il indique la proéminence du référent aux yeux du locuteur. (...) Autrement dit, l'article est un moyen de focaliser l'attention sur un référent. (...) C'est ainsi la trace de la subjectivité du locuteur dans le discours. »

À partir de ces quelques éléments définitionnels, lors et alors médiévaux seraient donc essentiellement anaphoriques ${ }^{25}$ : ils montrent rétroactivement qu'un cadre de discours s'est constitué, que certains éléments d'une situation ont été évoqués. La présence de le, dans lors comme dans alors, marque, de fait, qu'il y a du déjà énoncé, et ce faisant, donne à la phrase ou à l'élément de phrase qui précède le statut de posé explicite. Le nom heure, dont le référent -nous l'avons montré - peut avoir une extension vague, marque, de son côté que l'extension référentielle de lors et alors est largement définie par le contexte. Aussi n'est-il pas toujours aisé de déterminer ce à quoi lors ou alors servent de repère et, de ce fait, ils sont susceptibles de se trouver dans la zone dite annexe :

(9)Se la cité fust lors assise 
Ou alumee ou toute esprise,

Ne cuit que plus em plorissant

Ne greingnor deul en feïssant. (Roman de Thèbes, v. 1975-1978 ; vers 1150)

(10)« Beau Dieux ! Que le roy de Castille fu joieux de ceste nouvelle ! Il n'eust a fin souhet demandé mieulx ; il dist alors tout haultement : «Loé soit Dieu de ce beau secours(...) (Roman du Comte d'Artois : 68 ; vers 1453-1467)

ou occuper la place de fondement ${ }^{26}$, qu'ils soient précédés d'un coordonnant :

(11)Aprés souper, quant les napes furent levees, li rois semont ses chevaliers d'aler chacier en la forest de Kamaalot a l'endemain matin. Et lors dist Lancelos au roi : " Sire, vos m'avroiz a compaignon en ceste voie. (Mort le Roi Artu : 113 ; 1230)

(12)C'est pou de chose des houseaulx, dit monseigneur ; mais non pourtant, puis qu'il vous plaist, il seront ostez. « Et alors il abandonna sa prinse et se siet dessus l'erbe, et tend sa jambe ; et la belle fille luy oste l'esperon et puis luy tire l'un de ses houseaux, (...) (Cent Nouvelles nouvelles : 157 ; vers 1456)

ou apparaissent à l'initiale absolue de l'énoncé :

(13)Ele l'esgarde, bien l'entent,

Vers lui se trait et mot ne dit.

Lors se tormente, lors s'ocit. (Narcisse, v. 976-978; vers 1165-1175)

(14)Trestout l'ost arrivoit a ce lez ou l'escarmouche estoit. Alors fu le messaige mis hors parune autre porte, et passe tout par derriere l'ost, au trait d'un arc, que oncques ne fu apperceuz, et chevauche grant aleure vers son oncle, car moult lui tarde que il y puist estre pour dire ces nouvelles. (Jean d'Arras, Mélusine : 98 ; 1392-1393)

Du même coup, le lien anaphorique peut porter sur un contenu propositionnel équivalent à un seul membre de phrase ou à l'ensemble d'un paragraphe, qu'il appartient au lecteur (ou à l'interlocuteur) de repérer comme l'élément anaphorisé. La nature floue du lien anaphorique exprimé permet de relancer l'élan narratif ou encore de redonner une impulsion au récit ${ }^{27}$. Ceci apparaît assez clairement dans les énoncés suivants :

(15)(...) ; quant il s'entrencontrerent, lors veïssiez joie merveilleuse que li uns cousins fist a l'autre. Lors trest Boorz Lancelot a une part et li dist : " Avez vos encore oïes les noveles (...) (Mort le Roi Artu : 96 ; 1230)

(16)Alors le roy d'Angleterre commanda a l'ung de ses barons qu'il allast querir ung herault. Si fut incontinant venu, et alors le roy luy dit et chargea que il allast veoir celle belle compaignie et qu'il s'enquerist et demandast qui estoit le seigneur d'eulx, (...) (Roman de Jean de Paris : 28 ; 1494)

L'emploi de ces adverbes tisse en réalité une cohérence qui ne tient pas seulement à l'entité de la phrase ou du paragraphe anaphorisés, mais qui dépend aussi de la dimension de subjectivité qu'ils introduisent :

(17)Atant fine li parlemenz; si s'en revient li rois as tentes et enmeine avec soi la reïne. Lors commença entr'eus la joie si grant comme se Damledex i fust descenduz. (Mort le Roi Artu : $120 ; 1230)^{28}$

Ils chargent l'énoncé d'une certaine valeur affective, étymologiquement liée aussi bien à le qu'à heure. Ainsi peuvent-ils réaliser une transition entre l'extériorité de l'enchaînement narratif et l'intériorité du point de vue - d'où leur exploitation dans l'articulation très souple du discours au récit :

(18)Quant l'empereur vit la vaillance de cestes gens, et que l'un parti ou l'autre failloit que rompist, alors s'escria et dist : « Helas! Ou estoit mon cuer, de souffrir un tel inconvenient ? « Lors hastivement gecta sa flesche en disant : « Ho ! « Lors fust demandé dont il venoit et qu'il demandoit. (Antoine de la Sale, Jean de Saintré : $267 ; 1451)$ 
(19)Alors Madame lui dist : " Abbé, de tresbon cuer nous vous en remercions, aussi s'il estoit chose que pour vous nous puissions faire et pour tout le couvent, de tresbon cuer l'acomplirons, " alors Madame demanda a veoir les reliques. (Antoine de la Sale, Jean de Saintré : 246 ; 1451)

21 À la lumière de ces faits, il y a donc dans lors et alors d'une part une valeur anaphorique, d'autre part une valeur subjective, ce qui nous semble rendre compte d'une façon simple, globale et cohérente des propriétés communes des deux adverbes. Est-ce à dire qu'il s'agit de formes concurrentes, employées dans les mêmes contextes et avec les mêmes valeurs, ou est-il possible de définir des traits spécifiques pour alors?

\subsubsection{Propriété spécifique à alors}

À première vue et à la lecture d'un énoncé tel que :

(20)Alors le petit Saintré, tout honteux, le viz de honte tout enflamé, soy inclinant, avec les autres devant se mist. Et quant Madame le vist devant alors chemina tout en riant avec ses femmes et leur dist : "Mais que soions a la chambre, nous rirons". Lors dist dame Jehanne : "Ma dame, de quoy ?" (...) Madame le vist a genoulz elle lui dist: "Vous demeurrez, maistre, vous n'estes pas au compte des hommes de bien. Je veul cy parler a vous". Et alors la porte fut close. Madame, assise sur les piés du petit lit, le fist entre elle et ses femmesvenir, et lors print la foy de lui de ly dire de toutes ses demandes la verité.(Antoine de la Sale, Jean de Saintré : 6-7 ; 1451)

Il semblerait que lors et alors se trouvent en variation libre. On a ainsi toujours considéré que « (...) lors et alors sont souvent utilisés avec la même valeur sémantique. "(Sakari, 1997 : 352). Mais de fait, et de quelque manière qu'on les raccorde, cela n'entame pas l'évidence même selon laquelle alors apporte quelque chose de plus ${ }^{29}$.

\section{a. Opérations spécifiques introduites par la préposition à}

Nous ne pouvons, naturellement, envisager de renseigner sur l'ensemble des connaissances mises au jour par l'abondante littérature qui est consacrée à la préposition à. Mais il est à noter qu'on s'accorde en général - aussi bien traditionnellement que dans les travaux linguistiques plus récents - à reconnaitre son caractère ponctuel. Guillaume (1919) considère que nous retrouvons dans tous les emplois de la préposition à l'idée d'une ponctualité. Plus récemment, Berthonneau (1989 : 350) admet que « à apparaît comme un opérateur de discret ou de discrétisation ${ }^{\prime 30}$, ce qui est une autre manière de formuler le consensus sur son caractère ponctuel. Dans cette perspective, cette préposition exprime une référence temporelle stricte ou est associée à des prédicats de localisation temporelle, comme le souligne Borillo (1983, 1998 $)^{31}$. Il est un autre point sur lequel tout le monde s'accorde. C'est la valeur dynamique de la préposition à. Guillaume (1919 : 253) a été, à notre connaissance, le premier à le noter. Il considère qu'elle " est de forme linéaire ", c'est-à-dire qu'elle " représente tous les points d'une ligne de direction jusqu'au pointfinal ». Kemmer \& BatZeev Shyldkrot $(1996: 367)$ ont repris cette idée :

"A has a dynamic sense in which it expresses the trajectory of the figure to the point represented by the ground. Thus, both a path and a destination point or endpoint are invoked. »

Marque-Pucheu (2008: 88) affirme de même :

« (...) à indique le positionnement d'une entité par rapport à une autre ou par rapport à un procès (état, événement, activité, etc.), la première servant le plus 
souvent de point de départ et la seconde d'aboutissement, instaurant ainsi une dynamique. »

\section{b. L'opérativité32 de la préposition à} une prédominance si nette de l'idée de ponctualité. Cette propriété a un impact non seulement sur le sens de l'adverbe ainsi construit, mais aussi sur ses combinatoires. Alors a, de fait, un comportement en tout point semblable à celui du complément introduit par la préposition à : l'effet produit sur le lexème verbal et le temps grammatical ne diffère que sur des points de détail de celui que provoque le complément prépositionnel. Les lexèmes verbaux courants dans les phrases où apparaît alors sont - semble-t-il - des lexèmes perfectifs. Aussi, le temps grammatical le plus fréquent est le passé simple ${ }^{33}$. Il se dégage ainsi dans alors une interférence complexe entre le domaine lexical (nom de mesure de temps), le déterminant défini et la préposition $\mathrm{a}^{34}$. De là un ingrédient sémantique que l'on va retrouver dans tous les emplois de alors que sous la forme d'un sème, qui le distingue fondamentalement de lorsque. Mais avant d'aborder cette question, il nous faut souligner un aspect très important que notre analyse ait laissé jusqu'à'ici dans l'ombre. Lors et alors ont une attache privilégiée avec le mot que dont il complète la "morphologie intégrée ». Cette observation nous paraît féconde dans la mesure où elle met en évidence un fait banal, mais que l'on a parfois tendance à oublier, à savoir que ce sont des formes incomplètes ${ }^{35}$. Nous sommes donc conduite à aborder l'examen de la nature de leur complément. Nous entrerons ainsi dans le domaine du mot que. Le problème catégoriel que pose ce mot est le point central à partir duquel notre réflexion se développera.

\subsection{Le rôle fonctionnel de que ${ }^{36}$}

\subsubsection{Qui est que ?37}

Du point de vue morphologique, que est clairement un mot qu-. Reste à élucider ce qui dans son fonctionnement, peut le poser en affinité avec les autres mots qu-. Voici ce qu'affirme Touratier (1980: 75) :

« (...) qu- est le support formel et du morphème de subordination et du morphème anaphorique et correspond par conséquent à un amalgame de ces deux morphèmes.

$»$

Retrouve-t-on ces caractéristiques dans que, qui entre dans la composition des locutions conjonctives $?^{38}$ Ces deux propriétés corrélées semblent caractériser que dès les états de langue les plus anciens, même si son fonctionnement a évidemment subi évolutions et transformations au cours du temps ${ }^{39}$. Comme les autres mots $q u-$, il apparaît, de fait, à l'intersection de deux propositions et semble avoir la fonction de « subordonnant ». L'emploi de que dans :

(21)Que que ele se demante ensi,

Uns chevaliers del bois issi,

Qui de roberie vivoit (Chrétien de troyes, Erec et Enide, v. 2791-2793 ; vers 1170)

(21)Quant qu'estoient en la champaigne,

Si leur vint o grant compaigne

Richart li dus des Borgueignons. (Livre des Miracles de Notre-Dame de Chartes, v.

182-184 ; cité par Imbs (op. cit. : 299))

(22)U que il voit Aiol, si l'en araisona (Aiol, v.5933 ; fin du XIIe siècle)

Revue de Sémantique et Pragmatique, 38 | 2015 
onne une bonne illustration. Il est bien évident que son rôle fonctionnel est celui de la conjonction ${ }^{40}$ des deux éléments phrastiques. Que est aussi un anaphorique, ce qui lui permet de jouer un rôle vicaire ${ }^{41}$. Les données diachroniques nous permettent de vérifier le bien-fondé de cette allégation. Dès l'ancienne langue, il reprend, de fait, régulièrement quand et comme en proposition coordonnée :

(23)Quant che vint l'endemain par matin et que chil de le ville seurent que liempereres se estoit fuis, si ne font il mais el, si vienent il as portes. (Robert de Clari, Conquête de Constantinople : 70 ; après 1205)

(24)Pour venir à mon propoz de ceste armée d'Estampes, comme tous eussent souppé, et qu'il y avoit largement gens, se promenant par les rues (Philippe de commynes, Mémoires : 35 ; vers 1489-1498)

Cette aptitude anaphorisante tient à une indétermination sémantique basique, ce qui donne à penser que que est en fait un indéfini que vient instancier, saturer un termehôte ${ }^{42}$. En l'occurrence d'ailleurs, que représente anaphoriquement lors et alors dans l'élément phrastique qu'il introduit. Que épouse ainsi une double fonction, conforme à la fois à la valeur basique d'indétermination et à la double prédication qui le caractérise en propre ${ }^{43}$. Il exige la présence d'une suite textuelle dans son antécédence. Il a pour fonction d'assurer la connexion entre cet antécédent ${ }^{44}$ textuel et l'énoncé auquel il est incident. Il fonctionne comme une unité à double face connective : il est tourné cataphoriquement vers l'avant, et annonce donc la survenue d'un procès nouveau, et, en même temps, il se trouve aussi tourné en quelque sorte vers l'amont, puisqu'il fonctionne en corrélation avec l'élément de base lié au prédicat principal. Le point clé, pour ce qui nous occupe ici, est que, par son interdépendance avec lors ou alors, qui le tournent vers l'arrière, il opère une jonction avec l'amont et peut servir ainsi de plaque tournante discursive, réalisant alors pleinement la fonction d'un élément intermédiaire - qui provient de son ambivalence informationnelle, à la fois rétrospective et prospective. Avec lors et alors, qu'il présente sous une forme nouvelle, il contribue très fortement à la cohésion phrastique.

\subsubsection{La synergie de lors, alors et de que}

Nous l'avons constaté, le mot que a un sémantisme indéfini qui demande à être saturé par le cotexte. Aussi fonctionne-t-il en coalescence avec lors ou alors, qui lui confère un statut informationnel. L'indétermination du mot que se trouve ainsi spécifiée. Il ne peut exister lui-même sans lors ou alors mais il y est tout autant indispensable. On peut d'abord remarquer que lors ne peut se dispenser du que. Il a disparu de la langue, en tant que forme adverbiale autonome ${ }^{45}$. Alors est élargi par que $P$. Le que semble y intégrer l'apport du syntagme verbal qu'il nominalise. Au lieu que alors se réfère à la partie du message déjà énoncée, il situe ainsi le verbe principal grâce à la référence au procès de la subordonnée. On discerne alors une certaine forme d'interdépendance qui n'est pas seulement forte d'un point de vue morpho-syntaxique mais s'impose aussi sur le plan sémantique. Chacun des composants dépend pour son interprétation du cadre spécifiant apporté par l'autre élément avec lequel il est mis en relation. Ainsi en :

(25)Il faut jusques au bout courir à mon dommage, Or sus avecques moy retourne à Rothomage, Et là si tu fais voir à mes yeux esbahis Que tu sois Lidias, que ce soit ton païs, Qu'Amerine s'accorde à devenir ta femme, Chose impossible à croire à moins que d'estre infame, Je consens qu'espousez et comblez de plaisirs Vostre Hymen donne fin à vos justes desirs : Mais si ta fourbe esclatte et paroist tout nuë, Je veux lors en quittant cette Dame inconnuë Le ruisseau de mes pleurs d'oresnavant tary, 
Qu'à l'instant Ligdamon devienne mon mary. (Scudéry (de), Ligdamon et Lidias ou la Ressemblance : 86-87, 1631)

(26)Qui veut voir disputer cette matiere doctement, qu'il lise l'Apologie du Roy,composee par M.Cahyer, estant lors Ministre de Madame. (Aubigné (d') Confession catholique du sieur de Sancy : 264, 1630)

(27)Les manes estonnez le croiront un Alcide, Et, lors que ce guerrier viendra pour passer l'eau, Caron ne l'oseroit attendre en son batteau. (Pichou, Les Folies de Cardenio :113; 1629)

(28)Lors qu'elles y arriverent, ce berger chantoit, et estoit tellement ravy en son imagination, qu'encores qu'elles fussent tout aupres de luy, si est-ce qu'il ne les appercevoit point. (Urfé (d'), L'Astrée : $17-18$; 1631)

il est clair que l'adjonction de que à lors en influence le sens. L'adverbe, ayant un point de référence implicite, ne peut à lui seul introduire une relation de simultanéité entre deux propositions. Comme l'explicitent les exemples suivants, le mot que contribue aussi à filtrer les relations que peut établir alors :

(164)Or comme pour bien faire, il ne faut pas escrire d'une façon qui soit vuide de fleurs de rethorique comme le vulgaire parle ordinairement, bien que son langage soit bon : aussi c'est la raison de fuir un autre extremité qui se treuve alors que l'on n' escrit pas un mot que sous les voiles des figures :parce que l'obscurité se treuve tousjours en un discours lors qu'il est figuré par tout, et que rien n'y paroit de simple et naï, et mesmes alors que lesfigures ne sont pas bien appropriees, et qu'à tout propos on parle des fables poëtiques, et que l'on remplit ses vers d'une continuelle narration des noms et des avantures des dieux des anciens, et que par periphrases, ou autrement, on faict tousjours entendre dans un poëme : Parnasse, Helicon, Pegase, Pierides, Muses, Phebus, Apollon, Jupiter, Mercure, Amour, Cypris, Cytheree, Palas, Minerve, et les autres deïtez fabuleuses. (Deimier, L'Académie de l'art poétique, où sont vivement esclaircis et déduicts les moyens par où l'on peut parvenir à la vraye et parfaite connoissance de la poésie françoise : $280-281 ; 1610$ ) (165) Alors que tout flambant d'une lumiere sainte Il s'y sied en triomphe, et pompe, et majesté, L'univers se prosterne en reverence et crainte, Et nul ange n'en peut supporter la clarté. (Bertaut, Les OEuvres poétiques :31,1620)

(166) Mon ame en sa douleur est tellement confuse que ce qu'ore elle veut soudain elle refuse alors qu'elle le peut. (Urfé (d'), L'Astrée : 418 ; 1631)

En son absence, le contenu référentiel de alors devient plus diffus ou tend vers la fonction de « marqueur énonciatif de transition $»^{46}$ :

(167) Le berger alors se relevant, apres avoir fait une grande reverence, prit la parole de ceste sorte. ((Urfé (d'), L’Astrée : $47 ; 1631$ )

(168) Alors la bergere continua de ceste sorte : vous oyez, grande et sage nymphe, par la bouche mesme de Calidon, que s'il m'a aymée, je n'y ay contribué du mien, sinon d'estre telle que je suis, et contre cela, quel remede pouvois-je inventer? (ibid. : 59)

(169) (...) et par vostre foy, dit alors Silvandre, se faisant voir à l'impourveue, s'il vous en supplie, les luy accorderez-vous? (ibid. : 88)

En somme, que fait intervenir une force extérieure à l'entité dénotée parlors et alors, en interférant avec leurs propriétés internes et en intervenant directement sur leur nature. Que a donc un apport spécifique dans lorsque et alors que dont il fait partie. Sa participation n'est pas réduite à une dimension purement catégorielle. Il ne constitue pas simplement un appendice morphologique à lors et alors mais élargit également la fonction de ces adverbes dans le discours. 


\subsection{Valeurs de lorsque et alors que}

Le fonctionnement de lorsque et alors que ne semble pas déroger au fonctionnement général de leurs composantes. De prime abord, ils fonctionnent comme des anaphores. Leur envoi anaphorique peut être explicite comme dans :

(21) L'iver, lors que la nuit lente Fait au ciel si long sejour, Une vierge vigilenteS'éveilla davant le jour(...) (Ronsard, Quatrième livre des Odes : $378 ; 1550$ )

(22) Alda arriva un après-midi alors que Giono était seul dans la vieille maison de ses pères. (Bertheroy, Les voix du Forum ; cité par Borlé (1927 : 21))

Le lien anaphorique peut aussi être implicitement construit et renvoyer à une situation particulière :

(23) Les choses en étaient là lorsque la guerre éclata. (Chauve-Bertrand, La Question du calendrier : $106 ; 1920)$

(24) À son retour d'Alger, alors que je l'accueillais à l'aéroport, il m'a dit : « Ils ne l'emporteront pas au paradis! « II était plein de colère et d'amertume contre les agitateurs qui avaient organisé l'émeute. (Mendès-France, CEuvres complètes : 84-85 ;1990)

Lorsque et alors que présupposent, comme leurs adverbes de base, l'existence d'un contexte antérieur qui prépare ou amorce le procès qu'ils dénotent. Celui-ci intervient en tant qu'un moment-occurrence ${ }^{47}$ défini-identifié ${ }^{48}$, qui sert de cadre au procès principal :

(25) Lorsque le Roi, par haut désir, et cure Délibéra d'aller vaincre ennemis, Et retirer de leur prison obscure Ceux de son ost à grands tourments soumis, Il envoya ses fourriers en Judée Prendre logis sur place bien fondée (...) (Marot, L'Adolescence clémentine :272-273; 1538)

(26) Et telle je la vi sur le plus hault de la tour, alors que l'oyseau blanc (comme cy dessus je t'ay racompté) me tira l'esperit à un baiser, et l'eleva jusques là dessus. (Aneau, Alector ou Le Coq : histoire fabuleuse, t. $1: 82 ; 1560$ )

ou en tant qu'un moment possédant une certaine propriété :

(27) Jamais la vérité n'entre mieux chez les rois Que lorsque de la fable elle emprunte la voix. (Boursault, Ésope à la cour :119;1702)

(28) Sous le glaive français, ainsi de l'Angleterre Les escadrons vaincus vont mesurer la terre, Alors que réveillant nos antiques débats,Leur jalouse valeur nous appelle aux combats. (Roucher, Les Mois : 168 ;1779)

Cependant, même dans ce cas, avec un moment-type, il y a toujours quelque chose de l'ordre de l'occurrence. En effet - et c'est là leur troisième grande particularité lorsque et alors que possèdent la capacité de transformer le procès qu'ils introduisent en un intervalle compact, une période indivise à l'intérieur de laquelle il est impossible de pénétrer d'où il ressort que leur espace temporel, actualisé par le verbe qu'ils introduisent, ne peut être perçu que comme un tout global, impénétrable à l'analyse ${ }^{49}$ :

(29) La mémoire du déluge étant encore si fraîche parmi les hommes, lorsque Noévivait encore, Dieu fit ses promesses à Abraham, et lorsque Sem vivait encore, Dieu envoya Moïse, etc. (Pascal, Pensées : 300-301 ; 1662)

(30) (...) une heure plus tard, alors que tout le château silencieux semblait mort, le baron sortit à pas de loup de sa chambre et s'en vint gratter à la porte de son amie. (Maupassant, Contes et nouvelles, t. $1: 812 ; 1882$ )

Toutefois, les points communs entre lorsque et alors que masquent une divergence sémantique, expliquant pourquoi la langue laisse coexister les deux locutions. Alors que met au premier plan une lecture perfective, accomplie, du processus auquel il renvoie. C'est un morphème de ponctualité, qui signifie l'atteinte d'une limite sans 
débordement, comme en atteste le sémantisme du verbe ou, plus largement, de l'ensemble verbe-objet, verbe-attribut, qui est de type statif, résultatif, et ne correspond pas à un prédicat d'action. Cela ressort clairement des exemples suivants :

(31) Et alors que ledit seigneur Thieri ce vit en tel estat, Dieu scet c'il fut couroucé et de despit il ruait son chapiaulx au visaige dudit Growes et s'en fuit. (Vigneulles, Les Cent Nouvelles nouvelles : $135 ; 1515)$

(32) Il m'a semblé qu'il y avait là une image de la tristesse de l'univers, la tristesse qui vient de ce que l'on existe, celle qui m'émouvait si profondément alors que j'étais enfant et que je regardais derrière une vitre se lever les étoiles.(Green, Journal : $21 ; 1940$ )

41 Ainsi traitent-ils d'une façon différente l'itération des procès ${ }^{50}$. Alors que met en relief la structure en parties d'une pluralité d'événements, ce qui implique une vision discontinue du thème temporel, et empêche de le qualifier globalement par la présence d'une habitude :

(33) Alors que tes devoirs tentoient ma liberté, Je t'ay pour t'obliger mille fois escouté, Je t'ay donné le temps de me faire tes plaintes, J'ay senty vivement tes profondes attaintes. (Malleville, OEuvres poétiques : $265 ; 1649$ )

En revanche, lorsque ne sert pas à poser une pluralité. Il impulse un mouvement fédérateur des parties au tout, pour offrir une "vue d'ensemble " de la pluralité. En d'autres termes, lorsque fonctionnerait comme un "rassembleur», qui globalise les événements dénotés, lesquels sont envisagés dans leur ensemble. L'itération permet alors de caractériser une période, en ce sens quecette interprétation peut être qualifiée d'habituelle :

(34) Lorsque venait le tour de Christophe, souvent il n'en restait que trois sur l'assiette, et sa mère n'était pas servie. (Rolland, Jean-Christophe : L'Aube : 41 ; 1904)

Par ailleurs, l'espace signifié par alors que étant ponctuel, aucun décalage n'est permis entre les deux verbes. Ce morphème ne peut être employé qu'en cas de coïncidence parfaite. Contrairement à alors que, néanmoins, lorsque peut marquer la précédence temporelle (fondée iconiquement sur la précédence logique) ${ }^{51}$ :

(35) Lorsque tout fut couchiez, ledict bourgeois en habit de femme, comme dit est, entra et trouva ce maistre chanoine en beau hocqueton de camelot, acoustrez comme ung petit esmerillon et qui faisoit tant du miste et de l'honneste que merveille. (Vigneulles, Les Cent Nouvelles nouvelles : $100 ; 1515$ )

En somme, il paraît que lorsque et alors que partagent la propriété d'être fondamentalement anaphoriques, de construire une localisation du procès qu'ils introduisent, coïncidant avec la période référentielle introduite par le procès principal. Le propre de alors que est d'opérer sur un point ou un intervalle référentiel non impliqué en intervalle sémantique ${ }^{52}$. Lorsque n'introduit pas d'opération particulière ${ }^{53}$. Cette brève analyse permet, par contraste, de souligner que les fonctionnements qu'affichent lorsque et alors que réactivent les valeurs originelles de leurs composantes. C'est l'analyse de la structure morpho-sémantique de ces locutions qui nous a mise sur la voie d'une explication. Il faut maintenant tenter de définir comment elles ont été utilisées, dans quelle mesure elles sont interchangeables ou se spécialisent $t^{54}$. De fait, l'approche unitaire ici proposée n'a pas pour but de figer lorsque et alors que dans le carcan d'une définition close, ni de tenter de dégager un sens central « prototypique » autour duquel s'articuleraient tous les autres mais plutôt de 
constituer le principe organisateur de leur variation et la spécificité de la mise en oeuvre des principes généraux qui sous-tendent cette variation.

\section{Représentations du sens de lorsque et alors que}

L'analyse que nous allons mener, à présent, tentera de ressaisir les métamorphismes qui sont au coeur des travestissements variables de lorsque et alors que. Notre propos sera de montrer que si chacune de ces locutions conjonctives possède un éventail particulièrement large d'emplois et d'effets de sens, ils s'allient tous à son invariance sémantique. L'enjeu comporte essentiellement de revenir, en priorité, sur deux modalités, logiques ou énonciatives, l'opposition et la cause, universellement admises comme consubstantiellement liées au temps, et de mettre en valeur la manière dont lorsque et alors que les mettent en place.

\subsection{Simultanéité temporelle explicitée et opposition implicite}

La relation entre les deux contenus propositionnels est sans conteste de type temporel en :

(36) Pitié d'elle, de ne l'aimer pas davantage, de ne trouver pas davantage de raisonsde l'aimer, - et qu'elle ne fût pour lui qu'une parmi d'autres, alors qu'il était le seul pour elle, - et de ce qu'elle croyait qu'il lui donnait, quand il ne pouvait pas le lui donner. (Montherlant, Pitié pour les femmes : 136 ; cité par Imbs (1973, t.2 : $606 ; 1936)$

(37) Je me suis vu à cet instant par hasard dans une glace, et je me suis trouvé extraordinairement beau, alors que je n'y voyais plus qu'un squelette depuis des mois. (Guibert, À l'ami qui ne m’a pas sauvé la vie : $259-260 ; 1990$ )

mais une valeur supplémentaire semble présente. Alors que fait - semble-t-il -cohabiter, en les associant dans un même cadre temporel, deux contenus propositionnels, qui sont normalement incompatibles ${ }^{55}$. La valeur temporelle se teinte ainsi d'une valeur adversative basée sur l'existence d'un décalage entre ce qui correspond à une norme (commune à plus d'un) et ce qui est effectivement validé56. De même en :

(38) Craindre ! repartit le père avec une sorte de chaleur ; craindre lorsqu'il y a des hommes en péril, et que je puis, leur être utile ! (Chateaubriand, Atala : 96 ; 1801)

(39) C'est qu'on se croit parjure, lorsqu'on n'est qu'abusé. (Musset, cité par Guilbert, Lagane \& Niobey (1986, t.4:3112)

lorsque semble articuler la représentation factuelle des procès (leur localisation temporelle) et leur représentation intersubjective, et marquer ainsi une opposition entre les procès simultanés. Assorties d'une négation syntaxique ou morphologique, ${ }^{57}$ lorsque et alors que finissent par désigner une notion conceptuelle d'opposition. La relation oppositive n'est pas une relation a priori, mais une implicitation, relevant de l'intention informative du locuteur : elle est dérivée par raisonnement à partir de la relation temporelle explicitée. La synergie entre lorsque et alors que, qui réintroduisent sur scène leur composante subjective et la négation, impliquant prise de position du locuteur ${ }^{58}$, combine les ressources propres de chacun. Nous sommes tentée de conclure à une double prise en charge par le locuteur, qui aboutit à l'attribution d'un sens oppositif à lorsque et alors que. 


\subsection{Simultanéité et causalité} partir d'une relation de simultanéité temporelle. Elle est fondée sur un acte de recognition intersubjectif. Dans un exemple comme :

(40) Allons, allons ! Maître, cher ami... lorsque tant de malheureux vous bénissent! (Bernanos, La Joie : 636; cité par Imbs (1983, t.10 :1370) ; 1929)

il semble que ce soit l'énonciation du procès subordonné qui rend celle du procès principal possible en alignant son contenu propositionnel sur un autre censé mieux connu et par conséquent tenu pour admis a priori du destinataire, exactement à la façon d'un " lieu " rhétorique. Il revient ainsi au domaine pragmatique de surajouter une nuance causale à la relation de simultanéité temporelle établie par lorsque. Ce qui est encodé par parce que en :

(41) Il y a des hommes qui pleurent lorsque leur maîtresse les a quittés parce qu'ils ne retrouveront plus, le soir, en rentrant dans leur chambre, ses bras ouverts et ses lèvres tendues qui avaient ce goût rouge des grands baisers d'amour. (Philippe, La Mère et l'enfant : $42 ; 1900$ )

est simplement une relation causale objective, lorsque impose, quant à lui, un certain niveau d'implication du locuteur, ce qui constitue sa coopération à l'interprétation de son environnement discursif. Il en est de même en :

(42) Comment les riches Parisiens peuvent-ils jouir gaiment de leur luxe, alors que, pour quitter la ville (...) ils doivent (...) traverser quinze kilomètres d'une banlieue sordide ? (Duhamel, Querelles de famille : 43 ; cité par Sandfeld (1977 : \$198); 1932)

Il semble que alors que introduise un repère intersubjectif, qui ne porte pas seulement sur la délimitation temporelle du procès, mais aussi sur la construction d'une deuxième situation point de vue. La validation de l'occurrence des procès est repérée temporellement, c'est-à-dire comme appartenant au plan factuel, mais elle est accomplie notionnellement (subjectivement ou plutôt intersubjectivement), ce que manifeste la modalité interrogative intrinsèquement argumentative et polyphonique ${ }^{59}$. La mise en place d'une relation causale, n'est pas inscrite sémantiquement dans lorsque et alors que, mais est due à leur interaction avec des opérations énonciatives bien précises. On a vu que c'est à la situation d'énonciation même qu'il est fait allusion dans les énoncés ci-dessus : elle laisse des traces dans le sens faisant du discours ainsi réalisé, un lieu, un espace, qui est désigné respectivement par les modalités interrogative et exclamative. On a vu, également, que lorsque et alors que ne sont pas un facteur subsidiaire dans l'imposition d'une perspective causale au noyau conceptuel de simultanéité. Chacun semble, par contre, en être partie prenante, et figure formatrice, tout en en restant une réalisation toujours remarquable. Somme toute, les perspectives oppositive et causale découlent d'un ordonnancement intersubjectif des procès, que lorsque et alors que contiennent potentiellement. Les traits qui les singularisent semblent constituer le fondement dans leurs différentes modulations.

\section{Conclusion}

Nous avons essayé de formuler, sur des bases empiriques solides et étendues, des hypothèses vérifiables, n'excluant ni le conceptuel ni le contextuel, ni le stable ni le variable, sur les propriétés de lorsque et alors que. Nous avons tenté de saisir leur 
instance invariante. Nous nous sommes aussi penchée sur leurs emplois contextuels. Nous espérons avoir montré, à l'issue de cette étude, que les propriétés morphosémantiques de lorsque et alors que trouvent leur motivation au plan discursif. Nous avons montré au fil des exemples que lorsque et alors que ont gardé les propriétés sémantiques des adverbes qu'ils furent originellement. Nous avons établi que, comme pour les adverbes dont ils dérivent, ils mettent en jeu des interprétations " basiques " grandement identiques. Nous avons aussi observé que et la différence séparant lorsque et alors que ressortit au clivage qui se fait entre lors et alors. Nous avons essayé de mettre en évidence, en outre, le fait que que entre en interaction avec les propriétés sémantiques de lors et alors, et fournit ainsi un second domaine filtrant le contexte. En effet, alors que lors et alors établissent des liens en amont (avec des constituants d'énoncés, des énoncés ou des ensembles plus vastes), lorsque et alors que, tout en entretenant des liens avec le contexte précédent, sont fondamentalement orientées vers l'aval du discours. Ils sont prédestinés à jouer un rôle dans la structuration du discours et donc à assumer, en plus de la fonction idéationnelle (ou représentationnelle), une fonction textuelle. Nous avons vu que l'anaphore fait figure de fil d'Ariane. Les différents éléments qui entrent dans la composition de lorsque et alors que attestent de sa centralité ; ils la créditent d'un statut de fondement. Ainsi, comme nous les décrivions, ce sont des opérateurs ouverts, de part leur formation même, sur plusieurs cibles. Ils sont susceptibles de façonner la visée énonciative du discours dans lequel ils s'ancrent. Mais ils semblent être aussi les lieux privilégiés d'une déformabilité opérée par les différents éléments d'un énoncé et, également, par le cadre discursif. Au terme de cette étude, nous pensons avoir montré que lorsque et alors que ne sont pas aussi figés que le terme de locutions le laisse entendre. L'unité de ces formes analytiques s'est trouvée ainsi brisée par la démarche que nous avons adoptée, qui est l'antithèse de la thèse habituelle. La valeur sémantique des locutions conjonctives s'est avérée, au vu de notre investigation, conditionnée par l'interconnexion d'une quantité de domaines, celles-ci faisant l'objet d'une élaboration.

\section{BIBLIOGRAPHIE}

Anscombre, J.-C. \& Ducrot, O., 1983, L'argumentation dans la langue, Bruxelles Mardaga.

Austin, J.-L., [1962] 1993, Quand dire c'est faire, traduit par Lane, G., Paris, Seuil.

Bat-Zeev Shyldkrot, H., 1987, « Quand, alors que et tandis que : un cas classique d'évolution-

sémantique » Romance Notes $n^{\circ} 28,1: 45-50$.

Ben Hamad, L., 2008, « Les locutions conjonctives en question(s) », Linx $n^{\circ} 59$, Les conjonctions en diachronie : parcours sémantiques, 83-93.

Ben Hamad, L., 2012, «L'émergence des locutions conjonctives de simultanéité en ancien français ", in Actes du troisième Congrès mondial de Linguistique française, Actes en ligne:

www.linguistiquefrançaise.org.

Revue de Sémantique et Pragmatique, 38 | 2015 
Ben Hamad, L., 2013, « Réflexions sur le statut du mot que dans les locutions conjonctives: changement et permanence? ", in Van Acker, M., Boutier, M.-G., Hadermann, P. (éds.), Variation et changement en langue et en discours, Mémoires de la Société Néophilologique de Helsinki, pp. 105-132.

Benveniste, E., 1966, Problèmes de linguistique générale, t.1, Paris, Gallimard.

Benveniste, E., 1974, Problèmes de linguistique générale, t.2, Paris, Gallimard.

Bertin, A., 1997, L'expression de la cause en ancien français, Genève, Librairie Droz.

Berthonneau, A.-M., 1985, « Niveaux et opérations dans la description des compléments temporels ", Langue française $\mathrm{n}^{\circ} 66,20-40$.

Berthonneau, A.-M., 1989, Composantes linguistiques de la référence temporelle. Les compléments de temps, du lexique à l'énoncé, thèse d'Etat, Paris VII.

Borillo, A. , 1983, « Les adverbes de référence temporelle dans la phrase et dans le texte », DRLAV $\mathrm{n}^{\circ} 29,109-131$.

Borillo, A., 1986, « La quantification temporelle : durée et itérativité en français », Cahiers de grammaire $\mathrm{n}^{\circ} 11,119-154$.

Borillo, A., 1998, « Les adverbes de référence temporelle comme connecteurs temporels de discours ", in Vogeleer, S., Borillo, A., Vetters \& Vuillaume, M. (éds.), 131-145.

Bougy, C., 2000, «Les connecteurs temporels et l'apparition de lors que dans la langue française ", Syntaxe et sémantique $\mathrm{n}^{\circ} 1: 39-78$, Caen, Presses universitaires de Caen.

Buridant, C., 2000, Grammaire nouvelle de l'ancien français, Paris, Sedes.

Cadiot, P., 1993, « De et deux de ses concurrents : avec et à », Langages n¹10, 68-106.

Cadiot, P., 1997, Les prépositions abstraites en français, Paris, Armand Colin.

Cervoni, J., 1991, La préposition, étude sémantique et pragmatique, Paris, Duculot.

Clas, A. \& Gross, G., 1998, « Classes de figement des locutions verbales », in Le Figement lexical, Mejri, S. et al. (éds.) : 11-18.

Corblin, F., 1995, Les formes de reprise dans le discours. Anaphores et chaînes de référence, Rennes, Presses universitaires de Rennes, collection Langue / discours.

Culioli, A., 1990-1999, Pour une linguistique de l'énonciation : opérations et représentations, 3 tomes, Paris, Ophrys. Denis, D. \& Sancier-Chateau, A., 1994, Grammaire du français, Le Livre de Poche.

Epstein, R., 1995, « L'article défini en ancien français : l'expression de la subjectivité », Langue française $\mathrm{n}^{\circ} 107,58-71$.

Foulet, L., [1930] 19903, Petite syntaxe de l'ancien français, Paris, Champion.

Franckel J.- J., 1986, « Alors - Alors que », Bulag n 13, 17-49.

Franckel J.- J., 1989, Étude de quelques marqueurs aspectuels du français, Genève, Droz.

Gaatone, D., 1981, « Conjonctions et locutions conjonctives en français », Folia Linguistica, n¹4, 1/2, Mouton Publishers, 195-211.

Gaatone, D., 1984, « La locution ou le poids de la diachronie dans la synchronie », Le Moyen français, 14-15: 70-81, CERES.

Garagnon, A.-M., Calas, F., 2002, La phrase complexe. De l'analyse logique à l'analyse structurale, Paris, Hachette Supérieur. 
Garnier, B., 1999, « Syntaxe de l'article et subjectivité littéraire dans le Conte du Graal (vv. 1301-3407) » L'information grammaticale $\mathrm{n}^{\circ} 81$.

Graeme Ritchie, R.-L. 1907, Recherches sur la syntaxe de la conjonction "que" dans l'ancien français depuis les origines de la langue jusqu'au commencement du XIIIe siècle, Paris, Champion.

Gross, G., 1988, « Réflexions sur la notion de locution conjonctive », Langue française n 77, 19-36.

Gross, G., 1997, « Du bon usage de la notion de locution », La locution entre langue et usages, 201-223, Martins-Baltar, Fontenay-aux-Roses, ENS éditions.

Gross, G.\& Prandi, 2004, La finalité : fondements conceptuels et genèse linguistique, de boeck. Duculot. Guillaume, G., 1919, Le problème de l'article et sa solution dans la langue française, Paris, Hachette.

Guimier, C., 2000, « Non-congruence et congruence : alors que vs tandis que, » Syntaxe et sémantique $\mathrm{n}^{\circ} 1,79-111$.

Guimier, C. \& Oueslati, L., 2006, « Le degré de figement des constructions “verbe +adjectif invarié" ", Composition syntaxique et figement lexical : 17-38.

Kemmer, S. \& Bat-Zeev Shyldkrot, H., 1996, " The semantics of "empty prepositions" in French ", in Cognitive Linguistics in the Redwoods, 347-389.

Kleiber, G., 1981, Problèmes de référence : Descriptions définies et noms propres, Paris, Librairie C. Klincksieck.

Kleiber, G., 1983, « Article défini, théorie de la localisation et présupposition existentielle», Langue française $\mathrm{n}^{\circ}$ 57, 87-105.

Kleiber, G., 1987, Du côté de la référence verbale. Les phrases habituelles, Berne, Peter Lang.

Le Goffic, P.1993, Grammaire de la phrase française, Hachette Supérieur.

Imbs, P., 1956, Les propositions temporelles en ancien français : la détermination du moment, Paris, les Belles Lettres, Publications de la Faculté des lettres de l'université de Strasbourg.

Marandin, J.-M., 1998, Grammaire de l'incidence, MS. http://www.llf.cnrs.fr/Gens/Marandin/indexfr.php.

Marchello-Nizia, C., 1985, Dire le vrai : l'adverbe "si" en français médiéval. Essai de linguistique historique, Genève, Librairie Droz.

Marque-Pucheu, C., 1996, « Les alternances prépositionnelles : des substitutions très contraintes ", Linx n³4-35 : 219-232, Hommage à Jean Dubois, Paris, Centre de recherches linguistiques de Nanterre.

Marque-Pucheu, C., 2008, « La couleur des prépositions à et de », Langue française $\mathrm{n}^{\circ}$ 157, 74-105.

Martin, R., 1971, Temps et aspect, essai sur l'emploi des temps narratifs en moyen français, Klincksieck, Paris.

Ménard, P., 1998, Syntaxe de l'ancien français, Bordeaux, Bière.

Moignet, G., 1973, Grammaire de l'ancien français, Paris, Klincksieck.

Muller, C., 1991, La négation en français. Syntaxe, sémantique et éléments de comparaison avec les autres langues romanes, Genève, Librairie Droz.

Olsson, L., 1971, Étude sur l'emploi des temps dans les propositions introduites par « quand » et « lorsque » et dans les propositions qui les complètent en français contemporain, Uppsala : Acta Universitatis Upsaliensis 
Sakari, E., 1997, « Observations sur quelques adverbes de temps (or, lors, alors) en moyen français ", Le moyen français, philologie et linguistique, Approches du texte et du discours, 351-368.

Skårup, P., 1975, Les premières zones de la proposition en ancien français, Études romanes de l'université de Copenhague.

Skrélina, L. \& Čebelis, D., 1972, « La détermination du nom en ancien français», Romania, t.93, 289-302.

Stein, D. \& Wright, S., 1995, Subjectivity and subjectivisation, Linguistic perspectives, Cambridge, Cambridge University Press.

Stroumza, K. , 2002, Temporalité de l'énonciation et cristallisation discursive. Analyse sémantique de quelques différences entre "quand" et "lorsque", Thèse de doctorat, Paris, École des Hautes Études en sciences sociales.

Vaugelas (De), C.-F., 1647, Remarques sur la langue françoise, Paris.

Wilmet, M. , 1997, Grammaire critique du français, Duculot.

Bloch, O. \& Wartburg, W. (Von) , 2002, Dictionnaire étymologique de la langue française, Quadrige, Puf.

Dauzat, A., Dubois, J. \& Mitterand, H., 1994, Dictionnaire étymologique et historique du français, Paris, Librairie Larousse.

Dubois, J., Giacomo, M., Guespin, L., Marcellesi, C, Marcellesi, J-P. \& Mével, J-P., 2002, Dictionnaire de linguistique, Paris, Librairie Larousse.

Furetière, A., 1690, Dictionnaire universel, La Haye, Rotterdam, Arnout \& Reiner

Lerrs, 3 vol., (rééd. Paris, S.N.L. Dictionnaire Le Robert, 1978).

Gaffiot, F., [1934] 2000, Dictionnaire Latin-Français, Paris, Librairie Hachette.

Greimas, A.-J., 2001, Dictionnaire de l'ancien français, Paris, Larousse.

Imbs, P. (dir.), 1971-1994, Trésor de la langue française. Dictionnaire de la langue du XIXe et du XXe siècle (1789-1960), 16 vol., Paris, Editions du Centre National de la Recherche Scientifique (t.1-10), Gallimard (depuis le t.11)

Lafaye, B., 18845, Dictionnaire des synonymes de la langue française, Paris, Librairie Hachette \& Cie http://gallica.bnf.fr.

Wartburg, W. (Von), 1992), Französisches Etymologisches Wörterbuch, Eine Darstellung des galloromanischen sorachschatzes, Tübingen, Mohr/Basch, Hebing und Lichtenhan.

Base de Français Médiéval. Lyon : UMR5191ICAR/ENS-LSH, http://www.bfm.enslsh.fr Base du Dictionnaire de Moyen Français, UMR 7118 ATILF / Nancy 2. http://www.atilf.atilf.fr/ dmf.htm

UMR 7118 ATILF/Nancy2, http://www.frantext.fr

\section{NOTES}

1. Les idées ici avancées s'inscrivent dans une tradition de réflexion inaugurée par Gaatone (1984) et poursuivie par Gross \& Prandi (2004).

2. D'après Dubois, « le figement se caractérise par la perte du sens propre des éléments constituant le groupe de mots, qui apparaît alors comme une nouvelle unité lexicale, autonome 
et à sens complet, indépendant de ses composants "([1994] 2002 : 202). Cette attitude est partagée par plusieurs auteurs : « Dans les suites figées, le sens n'est pas le produit des éléments composants » (Clas \& Gross (1998: 11)). Cf. aussi Durieux (1998: 138) (« Le figement lexical est une unité lexicale autonome, dont la signification est complète et indépendante de ses composantes. (...) L'objet auquel réfère le figement résultant est indépendant des référents des morphèmes composants »).

3. Considérer les locutions conjonctives comme de simples variantes, ayant le même sens, aboutirait au sacrifice des nuances sémantiques que les conjonctions « simples ", vu leur grande polysémie, sont incapables de traduire et que la densité lexicale des locutions est capable, au contraire, de véhiculer.

4. Nous axons notre travail sur lorsque et alors que mais la plupart des phénomènes abordés ici sont aussi valables pour toutes les locutions conjonctives.

5. Le terme de globalisation sémantique est souvent utilisé pour désigner la non-compositionnalité des suites opaques et figées (cf., par exemple, Guimier \& Oueslati (2006 : 29)). Pour notre part, nous retiendrons ce terme pour montrer que l'on peut, tout au contraire, calculer le sens " global » des locutions conjonctives à partir du sens de leurs composantes.

6. Si les locutions lorsque et alors que ont donné lieu à plusieurs études, centrées sur leurs propriétés syntaxiques ou sémantiques ou encore sur certaines de leurs valeurs énonciatives, peu de travaux intègrent la description des adverbes dont ils dérivent dans leur champ descriptif, ne fût-ce que pour en tirer des éléments d'argumentation pour le domaine connexe qu'ils traitent.

7. L'unanimité est loin d'être faite sur ce qu'il est légitime de considérer comme une locution conjonctive. Encore faut-il reconnaître que la démarcation entre locutions conjonctives et conjonctions simples n'est pas toujours nette. Les limites qui les séparent sont instables et varient considérablement à tel point que certains considèrent lorsque, puisque des conjonctions simples (cf., par exemple, Denis \& Sancier-Chateau (op. cit. : 143) et Garagnon \& Calas (2002 : 75)) et d'autres rangent quand, comme et si parmi les locutions conjonctives (cf., par exemple Gaatone (1981 : 206) et Wilmet (1997 : 547)). Nous n'entendons pas ici juger du bien-fondé théorique de l'ensemble de ces assomptions. À l'issue de cette étude, c'est la notion même de locution, qui se trouvera placée sous un nouvel éclairage.

8. C'est notamment le cas de Martin \& Wilmet (1980 : § 320 et 68 ) qui établissent une partition entre les adverbes orcentriques qui présupposent l'actualité (or, hui, aujourd'hui, (h)ier, devant hier, l'autr'ier, (de)main, enuit) et les adverbes lorcentriques qui, par contre, ne présupposent pas l'actualité mais un repère quelconque : (lors, alors, la veille (ou le jor devant), l'endemain). Moignet (1988 : 282) énonce de même qu'il existe deux registres, celui du moment de la parole et celui du moment du passé pris comme référent. Sur la base de cette opposition fondamentale, l'auteur établit aussi deux listes. Lors apparaît, également encore, à côté de la veille (ou le jor devant), l'endemain..., signifiant le moment passé de référence dans un récit, par opposition à or, hui, hier, l'autrier, main, enuit ..., signifiant le présent, le passé ou le futur du locuteur.

9. Ce terme est de Benveniste (1966-1974). Rappelons que, dans sa perspective, la notion de récit (toute narration impersonnelle) s'oppose à celle de discours (une énonciation supposant un locuteur et un auditeur).

10. Cf. aussi Marchello-Nizia (1985 : 40-41), Sakari (1997 : 361-367), Buridant (2000 : 521-522).

11. Bloch \& Wartburg $(2002: 374,446)$ précisent, à leur tour, que or (en outre ore, ores) est issu d'un syntagme nominal à l'ablatif : ha hora, altération du latin classique hac hora 'à cette heure' et donnent lors (en outre lor et lores), « dont le développement a été parallèle au type qui a donné naissance à or ", comme équivalent de illa hora `à cette heure-là'. Greimas (1989) ainsi que Dauzat, Dubois \& Mitterand (1994) adoptent une attitude similaire. 
12. De l'avis général, l'apparition de alors (ou alores) est un phénomène secondaire, tardif, et elle résulte d'un fait d'analogie formelle avec adonc, atant, aluec (ou alues). On admet qu'une tendance à l'expressivité aboutit à la création de ces formes, parallèlement à celle de ilors (ou ilores, ylors), idonc (ou idunc) itant, itel, iluec et aussi itout à côté de lors, dunc, tel, tant, lues et tout.

13. Sur ce point, cf notamment Marchello-Nizia (2006:110).

14. On peut rappeler la remarque de Furetière ([1690] 1978) qui est, à ce titre, éclairante : Lors « vient de illa hora ».

15. Nous n'avons relevé aucune occurrence de a lors pour la période du français médiéval. Nous rencontrons, par contre, les formes non soudées $a$ tant et $a$ donc, à côté des formes soudées adonc et atant, comme en témoignent les énoncés suivants : Et a tant veez cy venir et entrer ses hommes de la garde. (Commynes, Le roman de Jehan de Paris : 77) ; Et atant s'en part tout esmerveillé, et s'en vient au palaix ou il fit son rapport au roy (...) (ibid. : 53) ; Et avoit à ce donc un homme à Gand, qui avoit estet brassères de mielz. (Froissart, Chroniques : 127) ; (...) si ques li pays et li royaumes d'Escoce estoit tous despourveus de bon conseil, pour aler ne resister contre les Englès, qui adonc estoient si poissamment entré en Escoce. (ibid. : 108)

16. Gaffiot ([1934] 2000 : 344) mentionne les formes ad horam et ad horas.

17. Ce n'est qu'en ancien français qu'apparaît cette nouvelle possibilité, l'article n'étant pas un morphème primitif. Les textes anciens marquent ainsi le début d'une évolution qui affecte la forme du syntagme origine.

18. Nous dénombrons 45 occurrences de a eure, 27 de a hore, 15 de a (à) heure et 3 de a ure. Notons que, pour l'ancien français, notre étude s'appuie sur le recours conjugué aux textes puisés à la base du BFM (la base du Français Médiéval) et à ceux dépouillés par Graeme Ritchie (1907), Nizia (1985), Imbs (1990), Bertin (1997) et Buridant (2000).

19. Nous renvoyons à Berthonneau (1989 : 470-492) pour un commentaire détaillé sur ce type de noms.

20. Cf. la définition proposée par Berthonneau (1985 : 26-27).

21. Glasser (1936) affirme, comme le note Imbs (op. cit. : 199), que l'homme du Moyen Age vit le temps avant de le penser, et il le vit d'une manière aussi proche que possible de l'expérience immédiate.

22. Un fort volume ne suffirait pas à décrire les travaux qui y ont été consacrés. (Cf. notamment Guillaume $(1919,1944)$, Kleiber $(1981,1983)$, Furukawa (1986), Corblin $(1987,1995))$.

23. En ancien français, l'article occupe, de fait, une place particulière. « [C'est] un déterminatif lexical et grammatical.» (Skrélina \& Čebelis (1972: 19))

24. Greimas (1989 : 335) indique aussi que l'article défini le est « proche, par le sens, du démonstratif du français moderne ».

25. C'est ce rôle d'anaphorique qui permet - on le verra - l'articulation des valeurs temporelles et notionnelles.

26. Nous empruntons les termes de zone annexe et place de fondement à Skårup (1975).

27. Certaines études ont essentiellement insisté sur le fait que ces adverbes introduisent dans le récit des événements un supplément de cohérence, de signification. Ils sont ainsi considérés comme des particules narratives qui contribuent à en constituer la cohésion et en assurer le flux. Ainsi Sakari (1997 : 361) écrit : « Situant le procès dans la chaîne des événements racontés, l'adverbe lors, comme alors ponctue le récit et en assure une certaine cohérence ".

28. Comme le remarque Ménard (1998: 183), à qui est emprunté cet exemple, « lors détache d'une manière dramatique un moment important de l'action ", à la différence de atant et adont qui « ont généralement une valeur assez neutre ».

29. Nous souscrivons pleinement à la position selon laquelle « la préposition apporte quelque chose de sémantique» (Moignet, 1974 : 283). 
30. Remarquons ici que Berthonneau ne manque pas de souligner que la sélection de à par un nom de mesure de temps (en l'occurrence le nom heure) résulte de son affinité propre avec cette opération sur le discret.

31. Aussi, employé dans des contextes où il exprime le lieu, à semble marquer que le lieu n'est envisagé que comme un point dans l'espace, sans considération de volume, le lieu pouvant être celui où l'on est ou celui où l'on va. De même, dans les compléments marquant la manière ou avec les compléments de moyen, il exprime toujours l'idée de ponctualité.

32. Ce terme est emprunté à Austin ([1962] 1993).

33. Cf. Martin (1971 : 201). Précisons que la requête de Martin est entreprise sur sept textes, de longueur variée : Froissart, Chroniques ; Jean Maillart, Le Roman du Comte d'Anjou; Joinville, Histoire de Saint Louis ; Bérinus ; Jean d'Arras, Mélusine ; Commynes, Mémoires ; Le Roman de Jehan de Paris.Notons que selon Berthonneau (1989

34. Notons que selon Berthonneau (1989 : 358) «La sélection mutuelle de à et de ledéterminant défini pour les noms du référentiel chronologique est une constante. ". Il est suggéré encore que : "La préposition à interdit le déterminant indéfini. » (Marque-Pucheu (1996:230))

35. Cette affirmation, somme toute assez largement partagée, prend toutefois une force nouvelle si l'on considère que les locutions conjonctives, et notamment lorsque et alors que ne présentent pas de figement complet dans leur structure.

36. Nous ne ferons qu'une incursion prudente dans ce domaine, qui est plus complexe qu'il n'y paraît et exige des investigations très poussées.

37. Tous les linguistes sont à peu près unanimes pour voir un spectre large de fonctionnements possibles pour le mot que. C'est la nature grammaticale du mot que qui est à la base de la controverse.

38. La réponse à cette question constituait la préoccupation majeure de Ben Hamad (2013). Nous reprendrons ici le résultat de sa démonstration sans évoquer le détail de l'argumentation.

39. Nous avons apporté les preuves de la justesse de ces observations. (Cf. Ben Hamad (2012) et (2013)).

40. Le terme de conjonction, légué par la tradition, est notoirement imprécis, comme le note fort justement Piot (1978: 17) : "Le mot conjonction a deux acceptions selon que l'on se rapporte au sens « verbal » ou au sens nominal de ce mot. Lorsque l'on parle de la conjonction de deux phrases, ou de deux phrases sous la conjonction, l'on fait référence à l'opération qui a pour résultat de conjoindre deux phrases. (Il s'agit donc là à proprement parler du sens « verbal » de ce mot). En revanche, lorsque l'on parle d'une ou des conjonction(s), il s'agit des éléments d'une classe grammaticale de mots invariables qui ont pour fonction de conjoindre deux phrases (et donc du sens «nominal » de ce mot). ». Nous l'utilisons ici dans la première perspective définie par Piot, c'est-à-dire dans son sens verbal.

41. Au XVIIe siècle, cette vicarisation par que paraît nécessaire. (Cf. Vaugelas (1647:62) : Il est beaucoup plus françois et plus elegant (...) de mettre que.»)

42. À la suite de Marandin (1998), nous appelons hôte l'élément syntaxique auquel un ajout est adjoint.

43. Cette double fonction intègre que d'une façon naturelle dans le schéma d'ensemble des termes en qu-. Comme le montre Muller (2008: 26) : «L'ensemble Qu- partage deux propriétés, l'indéfinition dans la sélection des individus d'un ensemble, et la contrainte de double prédication ». Dans le même esprit, Le Goffic (1993 : 41-42) avait précisé que : "Les termes en qu- (à valeur fondamentalement indéfinie) (...) ont (...) la propriété, d'une singulière importance de permettre d'enchâsser des structures de phrase comme termes de phrase "

44. Nous utilisons ici le mot antécédent dans son acception la plus générale et tout à fait littérale de « $^{\circ}$ précédent ${ }^{\circ} »$.

45. L'emploi absolu de lors est en principe exclu. D'un point de vue diachronique, c'est le résultat d'une évolution entamée dès l'ancien français. Sa décadence n'est cependant pas complète. Il s'emploie en couplage avec les prépositions dès et depuis, comme le note Borillo (1998:141). 
46. Cf. Molinier (2003).

47. Ceci expliquerait que le moment indiqué par lorsque soit considéré comme plus précis que celui indiqué par quand. Cf. notamment Lafaye (1884) : «Quand est général, vague, (...) Au contraire, lorsque est précis, (...). Dans les propositions générales, (...) où on parle d'une manière absolue, indépendamment des cas ou des événements particuliers, quand est le seul mot qui convienne (...). Mais dans les propositions particulières, où il s'agi de ce qui s'est effectivement passé, où l'on raconte, c'est lorsque qui doit être préféré. ».

48. Vraisemblablement, ce fait est à rattacher à la spécificité sémantique du nom heure.

49. Ce trait n'est pas lié à un contexte spécial mais est une caractéristique sémantique inhérente à lorsque et alors que, qui résulte de la valeur même de l'article défini. «L'article défini, dit Kleiber (1986: 55), véhicule une présupposition existentielle d'un ensemble ».

50. Notre analyse rejoint celle de Borillo (1986) ou celle de Kleiber (1987 : 115) - inspirée de Vlach (1981), selon laquelle deux cas de figures doivent être distingués : " une pluralité donnée comme simple itération " et " une pluralité occurrentielle présentée comme une fréquence " "Une phrase simplement itérative, écrit Kleiber (ibid.), est une phrase qui présente une situation comme étant vérifiée à deux, trois,...plusieurs reprises à l'intérieur d'un intervalle temporel. (...) Une phrase fréquentative, au contraire, présente l'itération comme s'étendant sur tout l'intervalle temporel. L'itération n'est pas vrai dans l'intervalle, mais pour l'intervalle temporel, parce qu'il s'y ajoute un jugement fréquentatif qui ne se conçoit que par rapport à l'intervalle tout entier». Nous nous reportons aussi à l'opposition relevée par Ducrot (1979) entre plusieurs fois et souvent.

51. Il est intéressant de noter que l'antériorité s'accompagne ici d'adjacence.

52. Il semble bien évident, au vu de ce que nous avons exposé, que le trait qui singularise le fonctionnement de alors, constitue le fondement pour le développement de toute une série d'emplois de alors que.

53. C'est ce qui explique que la palette de ses emplois est beaucoup plus étendue.

54. Les travaux existants ne se soucient guère d'expliquer la nature de ces nuances.

55. On n'a pas affaire à deux procès discordants qui sont mis ensemble et qui donnent ensuite lieu à des procès simultanés, mais au schéma inverse. La "discordance " ou la "non congruence " est ainsi une conséquence possible des emplois de alors que - elle peut émerger en même temps que son emploi temporel - et non la source de ses emplois, tel que le développe Guimier (2000) à la suite de Franckel (1986 et 1989).

56. Dans ce type d'exemples, alors que semble pouvoir alterner avec mais. Toutefois, l'on doit remarquer que c'est du " mais-triangulaire " (où la conclusion visée par le procès subordonné s'oppose directement au procès principal) qu'il s'agit et non du " mais-rectangulaire» (où l'opposition se situe entre les conclusions visées par les deux procès).

57. Sur ce point, voir Muller (1991: 53-68).

58. Voir à ce propos Culioli $(1990, \mathrm{t} 1: 113)$.

59. Cf. sur ce point Anscombre \& Ducrot (1983 : 115-137).

\section{RÉSUMÉS}

Sur le prétendu figement des locutions conjonctives : le cas de lorsque et alors que Ce travail a pour objet de mettre en perspective la spécificité des locutions conjonctives, qui réside dans le principe sous-jacent à leur formation, dans leur morphologie propre, et par conséquent dans leur 
structure sémantique. Nous étudions la structure interne de lorsque et alors que et montrons qu'elles ne sont pas aussi figées que le terme de locutions le laisse entendre. L'unité de ces locutions conjonctives se trouve ainsi brisée par la démarche que nous adoptons, qui est l'antithèse de la thèse habituelle.

Sur le prétendu figement des locutions conjonctives : le cas de lorsque et alors que This paper aims at demonstrating how productive it is to scrutinize the formation, the morphology, and the semantic structure of conjunctive phrases. To achieve this goal, we tried to study the internal structure of lorsque and alors que and to show that they are not as fixed as the term phrases suggests.

\section{AUTEUR}

\section{LEÏLA BEN HAMAD}

Université de Sousse 